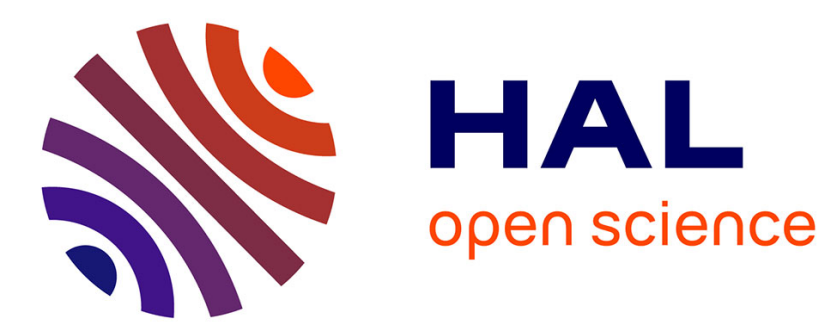

\title{
Polarisation électrique d'une interface par effet dispersif entre diélectriques anisotropes
}

\author{
Christian Girard, L. Galatry
}

\section{To cite this version:}

Christian Girard, L. Galatry. Polarisation électrique d'une interface par effet dispersif entre diélectriques anisotropes. Journal de Physique, 1983, 44 (11), pp.1273-1285. 10.1051/jphys:0198300440110127300 . jpa-00209712

HAL Id: jpa-00209712 https://hal.science/jpa-00209712

Submitted on 1 Jan 1983

HAL is a multi-disciplinary open access archive for the deposit and dissemination of scientific research documents, whether they are published or not. The documents may come from teaching and research institutions in France or abroad, or from public or private research centers.
L'archive ouverte pluridisciplinaire HAL, est destinée au dépôt et à la diffusion de documents scientifiques de niveau recherche, publiés ou non, émanant des établissements d'enseignement et de recherche français ou étrangers, des laboratoires publics ou privés. 


\title{
Polarisation électrique d'une interface par effet dispersif entre diélectriques anisotropes
}

\author{
C. Girard (*) et L. Galatry \\ Laboratoire de Physique Moléculaire (**), Université de Franche-Comté, 25030 Besançon Cedex, France
}

(Reçu le 28 avril 1983, accepté le 8 juillet 1983)

\begin{abstract}
Résumé. - On étudie la polarisation permanente d'origine dispersive au niveau d'une interface entre deux diélectriques, l'un isotrope, l'autre uniaxe. On considère comme point de départ que cette polarisation est due, comme pour les forces de van der Waals, au couplage entre les ondes électriques de surface du système et le champ issu des fluctuations locales (dipolaire et quadrupolaire) dans chaque élément de volume des solides en présence. De plus, dans cette approche, la polarisation est calculée dans le cadre de la théorie de la réponse non linéaire en utilisant pour chacun des milieux leurs hyperpolarisabilités par unité de volume et leurs constantes diélectriques. On effectue enfin une application numérique de la différence de potentiel résultant de cette polarisation permanente en modélisant les milieux constituant l'interface par des ensembles d'oscillateurs harmoniques isotropes et anisotropes.

Abstract. - The dispersive permanent polarization in the vicinity of the interface between two dielectrics, one of which is uniaxial and the other isotropic, is studied. This polarization is considered as arising (as for the van der Waals attraction) from the coupling between the electrical surface modes and the field due to the local dipolar and quadrupolar fluctuations in each of the volume elements of the interacting bodies. Moreover the polarization is calculated inside the frame of the non linear response through the hyperpolarizabilities per unit volume and the dielectric constants. Finally, one proposes a numerical estimation of the potential difference resulting from this permanent polarization by representing the constituting bodies by two ensembles of isotropic and anisotropic harmonic oscillators.
\end{abstract}

\section{Introduction.}

Les effets d'attraction entre systèmes matériels électriquement neutres (molécules ou solides macroscopiques) ont pour origine les fluctuations du champ électromagnétique. En effet l'attraction à longue distance (sans recouvrement des nuages électroniques) entre deux atomes peut ainsi être interprétée en terme d'interaction entre les dipôles instantanés et induits dans les deux systèmes $[1,2]$. De même la théorie de Lifchitz $[3,4]$ de l'attraction de van der Waals est fondée sur le fait que les fluctuations de polarisation de la matière créent des champs électriques pouvant être corrélés sur des distances macroscopiques. Diverses approches de ces effets s'appuyant sur la notion de modes de surface ont aussi été proposées [5-7]. Ces méthodes permettent de retrouver de façon plus directe les résultats de Lifchitz.

(*) Attaché de recherche au C.N.R.S.

(**) Equipe de recherche associée au C.N.R.S.
Par ailleurs des travaux récents ont montré que les fluctuations du champ électromagnétique sont également responsables, dans un couple d'atomes, d'une part, de l'apparition d'un moment dipolaire induit $[8,9]$ et d'autre part d'une anisotropie de la polarisabilité dynamique [10]. Il a aussi été suggéré [11, 12] que des fluctuations du même type engendrent une polarisation spontanée d'origine dispersive dans le cas d'une surface plane délimitant un milieu matériel isotrope.

Dans la première partie de ce travail, nous calculons la polarisation d'origine dispersive au niveau d'une interface plane constituée par deux milieux matériels de symétrie différente : l'un de symétrie globale $D_{\infty \mathrm{h}}$, l'axe de symétrie étant perpendiculaire à l'interface, l'autre isotrope. Ce type de géométrie a été choisi afin de simuler certains systèmes rencontrés en physicochimie des surfaces (par exemple des couches de molécules allongées disposées perpendiculairement à un substrat isotrope). Daus la seconde partie on effectue une estimation numérique 
de la différence de potentiel résultant de cette polarisation, en représentant les molécules du système par des oscillateurs harmoniques isotropes et anisotropes [13] suivant le milieu.

\section{Polarisation du système.}

Le cadre théorique utilisé dans ce travail sera celui introduit récemment par Richmond et Sarkies [14], pour calculer l'énergie potentielle d'interaction de deux molécules au voisinage d'une surface diélectrique. Cette approche a depuis été adaptée [15] à l'étude du moment dipolaire induit par physisorbtion dans un atome.

1.1 Présentation du système. - Considérons deux milieux continus (1) et (2) séparés par une surface plane $(z=0)$. Le milieu (1) anisotrope $(z>0)$, dont l'axe principal $C_{\infty}$ est perpendiculaire au plan de l'interface est caractérisé par les fonctions diélectriques $\varepsilon_{1 \|}(\omega)$ et $\varepsilon_{1 \perp}(\omega)$, et le milieu (2) isotrope $(z<0)$ par une fonction diélectrique scalaire $\varepsilon_{2}(\omega)$. Nous supposons de plus, comme dans la référence 15 , que tout le système est soumis à un champ électrique statique $F$ normal à la surface. Ainsi, si l'énergie potentielle d'interaction d'un échantillon $\mathrm{V}$ de volume unité, localisé en $R=\left(0,0, z_{0}\right)$, avec le reste du système, est égale à $U_{s}(R)(s=1,2$ suivant le milieu), alors le moment dipolaire de cet échantillon s'écrit :

$$
\mu_{s}(R)=-\nabla_{F} U_{s}(R)
$$

ou encore du fait de la symétrie cylindrique du système :

$$
\mu_{s}\left(z_{0}\right) \equiv \mu_{s}^{z}\left(z_{0}\right)=-\frac{\partial U_{s}\left(z_{0}\right)}{\partial F}
$$

et

$$
\mu_{s}^{x}=\mu_{s}^{y}=0 .
$$

Indépendamment du dipôle statique $\boldsymbol{\mu}_{s}\left(z_{0}\right)$, l'échantillon $\mathrm{V}$ présente des fluctuations de polarisation décrites à l'aide des dipôles et quadrupôles dépendant du temps $m_{s}(t)$ et $Q_{s}(t)$. Notons que ces fluctuations apparaissent à des fréquences $\omega_{i}\left(z_{0}\right)$, solutions d'une équation de dispersion $D\left(\omega, z_{0}\right)=0$, elles correspondent en fait aux fréquences des photons du champ électromagnétique échangés entre l'échantillon et le reste du système. Par conséquent, les multipôles $m_{s}(t)$ et $Q_{s}(t)$ seront développés en séries de Fourier :

$$
m_{s}(t)=\sum_{i} m_{s}\left(\omega_{i}\right) \mathrm{e}^{i \omega_{i} t} \quad \text { et } \quad Q_{s}(t)=\sum_{i} Q_{s}\left(\omega_{i}\right) \mathrm{e}^{i \omega_{i} t}
$$

La relation 2 montre que la connaissance de $\mu_{s}(R)$ est liée à la détermination de $U_{s}(R)$. Cette énergie est le travail qu'il faut fournir pour apporter l'échan- tillon V de $z= \pm \infty$ ( + pour le milieu (1), - pour le milieu (2)) à la position finale $\left(0,0, z_{0}\right)$; elle peut donc être identifiée à la variation de l'énergie libre de l'ensemble des modes de fréquence $\omega_{i}(z)$ lorsque l'on passe de la configuration $z=z_{0}$ à la configuration $z=\infty$. A la température $T$, on peut donc écrire [16] :

$$
\begin{array}{rl}
U_{s}\left(z_{0}\right)=k_{\mathrm{B}} & T \sum_{i}\left\{\log 2 \operatorname{sh}\left(\hbar \omega_{i}\left(z_{0}\right) / 2 k_{\mathrm{B}} T\right)-\right. \\
& \left.-\log 2 \operatorname{sh}\left(\hbar \omega_{i}(z=\infty) / 2 k_{\mathrm{B}} T\right)\right\},
\end{array}
$$

( $k_{\mathrm{B}}$ est la constante de Boltzmann).

Par ailleurs, la relation 4 peut être évaluée à partir de l'équation de dispersion du système $D\left(\omega, z_{0}\right)=0$ $[14,16]$, soit :

$$
U_{s}\left(z_{0}\right)=k_{\mathrm{B}} T \sum_{n=0}^{\infty}{ }^{\prime} \log D\left(i \xi_{n}, z_{0}\right) .
$$

Dans l'équation 5 la fonction $D\left(\omega, z_{0}\right)$ a pour zéros les fréquences $\omega_{i}\left(z_{0}\right)$ et pour pôles les fréquences $\omega_{i}(z=\infty)$ (le signe prime sur la sommation indique que le terme avec $n=0$ doit être multiplié par 1/2); de plus $\xi_{n}=2 \pi n k_{\mathrm{B}} T / \hbar$.

1.2 Polarisation du milieu anisotrope (1). Pour $z_{0}>0$ le milieu est supposé anisotrope (symétrie $D_{\infty \mathrm{h}}$ ). D'après les relations 2 et 5 , la polarisation $\mu_{1}\left(z_{0}\right)$ qui le caractérise s'écrit :

$$
\mu_{1}\left(z_{0}\right)=-k_{\mathbf{B}} T \frac{\partial}{\partial F} \sum_{n}^{\prime} \log D\left(i \xi_{n}, z_{0}\right) .
$$

Le calcul de $\mu_{1}\left(z_{0}\right)$ se réduit donc à la recherche de l'équation de dispersion $D\left(\omega, z_{0}\right)$ pour l'échantillon $\mathrm{V}$ (supposé à partir d'ici avoir un volume unité) en présence du champ statique $F$. La démarche utilisée pour accéder à cette équation introduit les étapes suivantes :

1.2.1 Potentiel électrique à l'intérieur du milieu (1). Dans le premier demi-espace $(z>0)$, le potentiel électrique au point courant $r(x, y, z)$ est écrit sous forme d'une somme sur les divers modes propres $i$ de contributions du type :

$$
\phi_{1}\left(r, \omega_{i}\right)=\phi_{1 \mathrm{v}}\left(r, \omega_{i}\right)+\phi_{1 s}\left(r, \omega_{i}\right) .
$$

La quantité $\phi_{1 \mathrm{v}}\left(r, \omega_{i}\right)$ représente la partie du potentiel électrique oscillant à la fréquence $\omega_{i}$, engendré par les charges situées dans l'échantillon V. Ce potentiel vérifie donc l'équation différentielle non homogène :

$$
\begin{aligned}
\nabla \cdot\left[\varepsilon_{1}\left(\omega_{i}\right) \cdot \nabla \phi_{1 \mathrm{v}}\left(r, \omega_{i}\right)\right] & = \\
& =-4 \pi \sum_{k} q_{k} \delta\left(r-r_{k}\left(\omega_{i}\right)-R\right)
\end{aligned}
$$

où $r_{k}$ est la position de la $k$-ième charge $q_{k}$ de l'échantillon V. 
Lorsque $|r-R|>\left|r_{k}\right|$ on peut développer le second membre de l'équation 8 en puissances de $r_{k}$, soit, en se limitant au $2^{\mathrm{e}}$ ordre :

$$
\begin{aligned}
& \nabla \cdot\left[\varepsilon_{1}\left(\omega_{i}\right) \cdot \nabla \phi_{1 \mathrm{v}}\left(r, \omega_{i}\right)\right]= \\
&=4 \pi\left\{\nabla \cdot \sum_{k} q_{k} r_{k}\left(\omega_{i}\right) \delta(r-R)\right. \\
&\left.-\frac{1}{2} \sum_{k} q_{k}\left(\nabla \cdot r_{k}\left(\omega_{i}\right)\right)^{2} \delta(r-R)\right\}
\end{aligned}
$$

(le terme d'ordre zéro est nul puisque le système est électriquement neutre).

Du fait de la symétrie du milieu (1) $\left(D_{\infty \mathrm{h}}\right)$ et du choix de l'axe principal parallèle à $O z$, le tenseur $\varepsilon_{1}$ a la forme suivante :

$$
\varepsilon_{1}^{x x}=\varepsilon_{1}^{y y} \equiv \varepsilon_{1 \perp}, \quad \varepsilon_{1}^{z z} \equiv \varepsilon_{1 \|}, \quad \varepsilon_{1}^{\alpha \beta}=0 \quad \text { si } \quad \alpha \neq \beta .
$$

Par conséquent, l'équation 9 s'écrit :

$$
\left\{\varepsilon_{1 \perp}\left(\omega_{i}\right)\left[\frac{\partial^{2}}{\partial x^{2}}+\frac{\partial^{2}}{\partial y^{2}}\right]+\varepsilon_{1 \|}\left(\omega_{i}\right) \frac{\partial^{2}}{\partial z^{2}}\right\} \phi_{1 \mathrm{v}}\left(r, \omega_{i}\right)=\left[m_{1}\left(\omega_{i}\right) . \nabla-\frac{1}{3} Q_{1}\left(\omega_{i}\right): \nabla \nabla\right] 4 \pi \delta(r-R)
$$

où $m_{1}\left(\omega_{i}\right)$ et $Q_{1}\left(\omega_{i}\right)$, introduits plus haut (équation 3 ), sont définis par

$$
m_{1}\left(\omega_{i}\right)=\sum_{k} q_{k} r_{k}\left(\omega_{i}\right)
$$

et

$$
Q_{1}\left(\omega_{i}\right)=\sum_{k}\left(q_{k}\left(3 r_{k}\left(\omega_{i}\right) r_{k}\left(\omega_{i}\right)-r^{2}\left(\omega_{i}\right)\right)\right)
$$

(I représente le tenseur $\delta_{i j}$ ).

Effectuons à présent le changement de variable :

$$
a=x /\left(\varepsilon_{1 \perp}\right)^{1 / 2}, \quad b=y /\left(\varepsilon_{1 \perp}\right)^{1 / 2} \quad \text { et } \quad c=z /\left(\varepsilon_{1 \|}\right)^{1 / 2} .
$$

L'équation 11 devient alors :

$$
\nabla_{\mathbf{\rho}} \phi_{1 \mathbf{v}}\left(\boldsymbol{\rho}, \omega_{i}\right)=\left[m_{1}\left(\omega_{i}\right) \cdot \tilde{\nabla}-\frac{1}{3} Q_{1}\left(\omega_{i}\right): \tilde{\nabla} \tilde{\nabla} \frac{4 \pi}{\varepsilon_{1 \perp}\left(\varepsilon_{1 \|}\right)^{1 / 2}}\right] \delta\left(\boldsymbol{\rho}-\boldsymbol{\rho}_{0}\right)
$$

avec

et

$$
\boldsymbol{\rho}=(a, b, c), \quad \boldsymbol{\rho}_{0}=\left(0,0, \rho_{0}=z_{0} /\left(\varepsilon_{1 \|}\right)^{1 / 2}\right), \quad \nabla_{\boldsymbol{\rho}}=\left(\frac{\partial}{\partial a}, \frac{\partial}{\partial b}, \frac{\partial}{\partial c}\right)
$$

$$
\tilde{\nabla}=\left(\frac{1}{\left(\varepsilon_{1 \perp}\right)^{1 / 2}} \frac{\partial}{\partial a}, \frac{1}{\left(\varepsilon_{1 \perp}\right)^{1 / 2}} \frac{\partial}{\partial b}, \frac{1}{\left(\varepsilon_{1 \|}\right)^{1 / 2}} \frac{\partial}{\partial c}\right) .
$$

L'équation 13 a pour solution [18] :

$$
\phi_{1 \mathrm{v}}\left(\boldsymbol{\rho}, \omega_{i}\right)=\left[-m_{1}\left(\omega_{i}\right) \cdot \tilde{\mathbf{v}}+\frac{1}{3} Q_{1}\left(\omega_{i}\right): \tilde{\mathbf{v}} \tilde{\mathbf{v}}\right] \frac{1}{\left|\boldsymbol{\rho}-\rho_{0}\right|} \frac{1}{\varepsilon_{1 \perp}\left(\varepsilon_{1 \|}\right)^{1 / 2}}
$$

Décomposons maintenant la fonction $1 /\left|\boldsymbol{\rho}-\boldsymbol{\rho}_{0}\right|$ en série de Weyl [18] :

$$
\frac{1}{\left|\rho-\rho_{0}\right|}=\frac{1}{2 \pi} \int \mathrm{e}^{i(r a+s b)} \mathrm{e}^{\left(r^{2}+s^{2}\right)^{1 / 2}\left(c-\rho_{0}\right)} \times \frac{\mathrm{d} r \mathrm{~d} s}{\left(r^{2}+s^{2}\right)^{1 / 2}}, \quad\left(c<\rho_{0}\right) .
$$

En substituant (15) dans (14), puis en posant $u=r /\left(\varepsilon_{1 \perp}\right)^{1 / 2}, v=s /\left(\varepsilon_{1 \|}\right)^{1 / 2}, l=(x, y), k=(u, v), \bar{\varepsilon}_{1}=\left[\varepsilon_{1 \perp}\left(\omega_{i}\right)\right.$ $\left.\varepsilon_{1 \|}\left(\omega_{i}\right)\right]^{1 / 2}, \delta=\left[\varepsilon_{1 \perp}\left(\omega_{i}\right) / \varepsilon_{1 \|}\left(\omega_{i}\right)\right]^{1 / 2}$ et $k_{1}=k \delta\left(\omega_{i}\right)$ il vient finalement :

avec :

$$
\phi_{1 \mathrm{v}}\left(r, \omega_{i}\right)=-\frac{1}{2 \pi \bar{\varepsilon}_{1}} \iint(M+1 / 3 Q) \mathrm{e}^{i k . l} \mathrm{e}^{k_{1}\left(z-z_{0}\right)} \mathrm{d} u \mathrm{~d} v
$$

$$
M=\frac{i}{k}\left[m_{1}^{x}\left(\omega_{i}\right) u+m_{1}^{y}\left(\omega_{i}\right) v\right]+m_{1}^{z}\left(\omega_{i}\right) \delta
$$

et

$$
Q=Q_{1}^{x x}\left(\omega_{i}\right) \frac{u^{2}}{k}+Q_{1}^{y y}\left(\omega_{i}\right) \frac{v^{2}}{k}-k \delta^{2} Q_{1}^{z z}\left(\omega_{i}\right)+2 Q_{1}^{x y}\left(\omega_{i}\right) \frac{u v}{k}-2 i\left[Q_{1}^{x z}\left(\omega_{i}\right) u+Q_{1}^{y z}\left(\omega_{i}\right) v\right] \delta
$$


La seconde contribution $\phi_{1 s}\left(r, \omega_{i}\right)$ à l'équation 7 représente le potentiel créé en $r$ par la présence pour $z \cong 0$ des modes de surface de fréquence angulaire $\omega_{i}$. $\phi_{1 s}$ vérifie donc l'équation homogène suivante [19] :

$$
\nabla \cdot\left[\varepsilon_{1}\left(\omega_{i}\right) \cdot \nabla \phi_{1 s}\left(r, \omega_{i}\right)\right]=0
$$

ou encore après changement de variable (cf. Eq. 12) :

$$
\nabla_{\mathbf{\rho}}^{2} \phi_{1 s}\left(\rho, \omega_{i}\right)=0 .
$$

La géométrie du système (présence d'une interface en $z=0$ ) autorise à choisir pour l'équation 19 une solution de la forme :

$$
\phi_{1 s}\left(\boldsymbol{\rho}, \omega_{i}\right)=\iint f_{1}\left(\omega_{i}\right) \mathrm{e}^{i(r a+s b)} \mathrm{d} s \mathrm{~d} r .
$$

Après substitution de (20) dans (19) on obtient :

$$
\phi_{1 s}\left(r, \omega_{i}\right)=\iint A_{1}\left(k, \omega_{i}\right) \mathrm{e}^{-k_{1} z} \mathrm{e}^{i k . l} \mathrm{~d} u \mathrm{~d} v(z>0),
$$

où $A_{1}\left(k, \omega_{i}\right)$ est l'amplitude d'un mode de surface monochromatique et où $k^{2}=u^{2}+v^{2}$.

Il semble important à ce niveau de faire une remarque. En effet, contrairement à la démarche de la théorie de van Kampen et al. [5], où l'introduction d'un champ macroscopique moyen est suffisante pour rendre compte des effets d'attraction entre deux surfaces planes, il est nécessaire dans la présente étude de prendre en compte le champ microscopique local $-\nabla \phi_{1 \mathrm{v}}$ engendré par les fluctuations propres de l'échantillon étudié. En effet pour accéder à des observables associées à un élément de volume du système (force de dispersion, moment dipolaire, ...), nous devons établir un lien entre les fluctuations de polarisation de cet élément de volume et les fluctuations du champ électromagnétique du reste du système (en particulier au niveau de la surface).

1.2.2 Potentiel électrique à l'intérieur du milieu (2). - Dans le second demi-espace $(z<0)$ et en analogie avec [14] seul le terme dû aux ondes de surfaces $\phi_{2 s}\left(r, \omega_{i}\right)$ sera introduit (réciproquement, lorsque l'on s'intéressera à la polarisation d'un échantillon $\mathrm{V}$ de volume unité appartenant à ce second milieu, il sera nécessaire d'y introduire un second terme $\phi_{2 \mathrm{v}}\left(r, \omega_{j}\right)$ dû aux fluctuations électriques à l'intérieur de l'échantillon lui-même). Dans le milieu isotrope $\phi_{2 s}\left(r, \omega_{i}\right)$ est solution de l'équation de Laplace :

$$
\Delta \phi_{2 s}=0
$$

et sera donc choisi sous la forme :

$$
\phi_{2 s}\left(r, \omega_{i}\right)=\iint A_{2}\left(k, \omega_{i}\right) \mathrm{e}^{i k . l} \mathrm{e}^{k z} \mathrm{~d} u \mathrm{~d} v \quad(z<0) .
$$

1.2.3 Conditions aux limites. - La continuité du potentiel et de la composante normale de l'induction au voisinage de la surface s'exprime de la façon suivante :

$$
\phi_{1 s}\left(x, y, 0 ; \omega_{i}\right)+\phi_{1 \mathrm{v}}\left(x, y, 0 ; \omega_{i}\right)=\phi_{2 s}\left(x, y, 0 ; \omega_{i}\right)
$$

$$
\begin{aligned}
\varepsilon_{1 \|}\left(\omega_{i}\right)\left\{\left.\frac{\partial \phi_{1 s}}{\partial z}\right|_{r=R}+\left.\frac{\partial \phi_{1 \mathrm{v}}}{\partial z}\right|_{r=R}\right\}= & \\
& =\left.\varepsilon_{2}\left(\omega_{i}\right) \frac{\partial \phi_{2}}{\partial z}\right|_{r=R}
\end{aligned}
$$

Une remarque paraît nécessaire au sujet de l'utilisation, dans les équations 24 et 25 , des conditions aux limites de l'électrostatique classique. On sait en effet que, dans le cas où la matière ne répond pas localement à une excitation électromagnétique, c'est-àdire lorsque les constantes diélectriques et, de façon plus générale, les susceptibilités non linéaires, dépendent d'un ou plusieurs vecteurs d'onde, alors les conditions de continuité du potentiel et de la composante normale de l'induction ont donné lieu à des discussions approfondies [16, 20]. On peut faire néanmoins la remarque suivante : bien que les potentiels électriques qui interviennent ici (cf. Eqs. 21 et 23) aient été développés en séries de Fourier spatiales, la réponse du milieu est toujours traitée de façon locale (ou encore sans dispersion spatiale) puisque les constantes diélectriques $\varepsilon_{1}$ et $\varepsilon_{2}$ ne dépendent que de $\omega$. Sur ce point le présent traitement est analogue à celui de la référence 5 dans lequel, pour obtenir l'attraction de van der Waals entre deux solides on applique directement les conditions aux limites de l'électrostatique élémentaire aux composantes de Fourier du potentiel (c'est-à-dire aux grandeurs $A_{1} \mathrm{e}^{i k . l} \mathrm{e}^{k z}$ et $A_{2} \mathrm{e}^{i k . l} \mathrm{e}^{-k z}$ ). Par ailleurs la présence, dans le calcul, de densités de quadrupôles (Eq. 28) et de gradients de champ (Eq. 27) fait implicitement référence à des effets de non-localité. Néanmoins la prise en considération de ces effets ne semble pas être de nature à invalider les conditions aux limites (Eqs. 24 et 25) puisqu'elle ne conduit dans le présent calcul à aucune des ambiguités sur les amplitudes des champs qui avaient suscité les travaux de la référence 20 .

A partir des relations 16, 21 et 23, on obtient :

$$
A_{1}\left(k, \omega_{i}\right)-\frac{1}{2 \pi \bar{\varepsilon}_{1}}(M+1 / 3 Q) \mathrm{e}^{-k_{1} z_{0}}=A_{2}\left(k, \omega_{i}\right)
$$

et

$$
\bar{\varepsilon}_{1} A\left(k, \omega_{i}\right)+\frac{1}{2 \pi}(M+1 / 3 Q) \mathrm{e}^{-k_{1} z_{0}}=\varepsilon_{2} A_{2}\left(k, \omega_{i}\right) .
$$

Ainsi, pour l'amplitude $A_{1}\left(k, \omega_{i}\right)$, il vient, après élimination de $A_{2}\left(k, \omega_{i}\right)$ entre les deux relations 26 :

$$
A_{1}\left(k, \omega_{i}\right)=\frac{1}{2 \pi \bar{\varepsilon}_{1}} \Delta \mathrm{e}^{-k_{1} z_{0}}(M+1 / 3 Q)
$$


avec

$$
\Delta=\left[\bar{\varepsilon}_{1}\left(\omega_{i}\right)-\varepsilon_{2}\left(\omega_{i}\right)\right] /\left[\bar{\varepsilon}_{1}\left(\omega_{i}\right)+\varepsilon_{2}\left(\omega_{i}\right)\right] .
$$

\subsubsection{Equations constitutives pour la polarisation $d u$} volume unité $V$ en présence d'un champ statique $F$. On peut maintenant considérer que les fluctuations des charges dans le volume $V$ sont induites par le champ $E_{1 s}$ des ondes de surface. Par conséquent, le dipôle et le quadrupôle $m_{1}\left(\omega_{i}\right)$ et $Q_{1}\left(\omega_{i}\right)$ peuvent être reliés par des équations constitutives à $E_{1 s}$ et à $F$. Dans un souci de cohérence, ces équations font intervenir les polarisabilités ${ }^{1} \tilde{\chi}_{1}^{1}$ et ${ }^{2} \tilde{\chi}_{1}^{1}$ et les hyperpolarisabilités par unité de volume ${ }^{1} \tilde{\chi}_{1}^{1+1},{ }^{2} \tilde{\chi}_{1}^{1+1}$ et ${ }^{1} \tilde{\chi}_{1}^{1+2}$ (ces grandeurs sont définies dans l'annexe $B$ ); d'où :

avec

$$
\begin{gathered}
m_{1}\left(\omega_{i}\right)={ }^{1} \tilde{\chi}_{1}^{1}\left(\omega_{i}\right) \cdot E_{1 s}+{ }^{1} \tilde{\chi}_{1}^{1+1}\left(0, \omega_{i}\right): F E_{1 s}+\frac{1}{3}{ }^{1} \tilde{\chi}_{1}^{1+2}\left(0, \omega_{i}\right): F \nabla E_{1 s} \\
Q_{1}\left(\omega_{i}\right)={ }^{2} \tilde{\chi}_{1}^{1}\left(\omega_{i}\right) \cdot E_{1 s}+{ }^{2} \tilde{\chi}_{1}^{1+1}\left(0, \omega_{i}\right): F E_{1 s}
\end{gathered}
$$

$$
E_{1 s}=\left[-\nabla \phi_{1 s}\left(r, \omega_{i}\right)\right]_{r=R} .
$$

Compte tenu de la symétrie du milieu (1) (cf. annexes A et B, Eqs. A.5, A.6, A.9 et B.4) et de la forme du champ $E_{1 s}\left(\right.$ Eqs. 21 et $\left.26^{\prime}\right)$, les relations 27 et 28 s'écrivent :

$$
\begin{gathered}
m_{1}^{\gamma}\left(\omega_{i}\right)=\frac{\Delta}{8 \bar{\varepsilon}_{1} \delta^{3}}\left[m_{1}^{\gamma}\left(\omega_{i}\right)\left(\frac{{ }^{1} \tilde{\chi}_{1 \perp}^{1}}{z_{0}^{3}}-\frac{{ }^{1} \tilde{\chi}_{1 \perp}^{1+2} F}{z_{0}^{4}}\right)+\frac{Q_{1}^{\gamma z}\left(\omega_{i}\right){ }^{1} \tilde{\chi}_{1 \perp}^{1}}{z_{0}^{4}}\right] \\
m_{1}^{z}\left(\omega_{i}\right)=\frac{\Delta}{4 \bar{\varepsilon}_{1} \delta^{3}}\left[m_{1}^{z}\left(\omega_{i}\right)\left(\frac{{ }^{1} \tilde{\chi}_{1 \|}^{1} \delta^{2}}{z_{0}^{3}}-\frac{{ }^{1} \tilde{\chi}_{1 \|}^{1+2} F\left(\delta^{2}+1 / 2\right)}{2 z_{0}^{4}}\right)-Q_{1}^{z z}\left(\omega_{i}\right) \frac{{ }^{1} \tilde{\chi}_{1 \|}^{1}\left(\delta^{2}+1 / 2\right)}{2 z_{0}^{4}}\right] \\
Q_{1}^{\gamma z}\left(\omega_{i}\right)=\frac{\Delta}{8 \bar{\varepsilon}_{1} \delta^{3}}\left[m_{1}^{\gamma}\left(\omega_{i}\right) \frac{{ }^{1} \tilde{\chi}_{1 \perp}^{1+2} F}{z_{0}^{3}}-Q_{1}^{\gamma z}\left(\omega_{i}\right) \frac{{ }^{1} \tilde{\chi}_{1 \perp}^{1+2} F}{z_{0}^{4}}\right]
\end{gathered}
$$

$\operatorname{avec} \gamma=(x$ ou $y)$.

$$
Q_{1}^{z z}\left(\omega_{i}\right)=-2 Q_{1}^{x x}\left(\omega_{i}\right)=-2 Q_{1}^{y y}\left(\omega_{i}\right)=\frac{\Delta}{4 \bar{\varepsilon}_{1} \delta^{3}}\left[m_{1}^{z}\left(\omega_{i}\right) \frac{{ }^{1} \tilde{\chi}_{1 \|}^{1+2} F \delta}{z_{0}^{3}}-Q_{1}^{z z}\left(\omega_{i}\right) \frac{{ }^{1} \tilde{\chi}_{1 \|}^{1+2} F\left(\delta^{2}+1 / 2\right)}{z_{0}^{4}}\right]
$$

et

avec

$$
Q_{1}^{y x}\left(\omega_{i}\right)=Q_{1}^{x y}\left(\omega_{i}\right)=0
$$

$$
{ }^{1} \tilde{\chi}_{1 \perp}^{1} \equiv{ }^{1} \tilde{\chi}_{1}^{1 x x}\left(\omega_{i}\right)={ }^{1} \tilde{\chi}_{1}^{1 y y}\left(\omega_{i}\right), \quad{ }^{1} \tilde{\chi}_{1 \|}^{1} \equiv{ }^{1} \tilde{\chi}_{1}^{1 z z}\left(\omega_{i}\right)
$$

et

$$
{ }^{1} \tilde{\chi}_{1 \|}^{1+2} \equiv{ }^{1} \tilde{\chi}_{1}^{1+2 z z z z}\left(0, \omega_{i}\right), \quad{ }^{1} \tilde{\chi}_{1 \perp}^{1+2} \equiv{ }^{1} \tilde{\chi}_{1}^{1+2 x z x z}\left(0, \omega_{i}\right)
$$

Les relations 30 permettent d'écrire un système linéaire et homogène par rapport aux variables $m_{1}^{x}, m_{1}^{y}, m_{1}^{z}$, $Q_{1}^{x z} / z_{0}, Q_{1}^{y z} / z_{0}$ et $Q_{1}^{z z} / z_{0}$. Ce système possède une solution non nulle si et seulement si le déterminant principal $D\left(\omega, z_{0}\right)$ qui lui est associé est nul. Cette condition permet d'écrire l'équation de dispersion recherchée $D\left(\omega, z_{0}\right)=0$ dont les solutions sont les fréquences $\omega_{i}$ possibles. Cette équation s'écrit, en ne conservant que les termes du premier ordre par rapport à $F$ :

$$
\begin{aligned}
D\left(\omega, z_{0}\right)=\left[1-\frac{{ }^{1} \tilde{\chi}_{1 \perp}^{1} \Delta}{8 \bar{\varepsilon}_{1} \delta^{3} z_{0}^{3}}+\frac{{ }^{1} \tilde{\chi}_{1 \perp}^{1+2} F \Delta}{8 \bar{\varepsilon}_{1} \delta^{3} z_{0}^{4}}\right]^{2}[1 & \left.-\frac{{ }^{1} \tilde{\chi}_{1 \|}^{1} \Delta}{4 \bar{\varepsilon}_{1} \delta^{3} z_{0}^{3}}+\frac{{ }^{1} \tilde{\chi}_{1 \|}^{1+2} F \Delta\left(\delta^{2}+1 / 2\right)}{8 \bar{\varepsilon}_{1} \delta^{3} z_{0}^{4}}\right] \times \\
\times & {\left[1+\frac{1+{ }^{1} \tilde{\chi}_{1 \perp}^{1+2} F \Delta}{8 \bar{\varepsilon}_{1} \delta^{3} z_{0}^{4}}\right]^{2}\left[1+\frac{{ }^{1} \tilde{\chi}_{1 \|}^{1+2} F \Delta\left(\delta^{2}+1 / 2\right)}{8 \bar{\varepsilon}_{1} \delta^{3} z_{0}^{4}}\right]=0 . }
\end{aligned}
$$

Après utilisation de la relation 6 , le moment dipolaire permanent acquis par l'élément de volume $V$ (de volume unité) s’écrit :

$$
\mu_{1}\left(z_{0}\right)=-\frac{k_{\mathrm{B}} T}{4 z_{0}^{4}} \sum_{n}^{\prime} \frac{\Delta\left(i \xi_{n}\right)}{\bar{\varepsilon}_{1}\left(i \xi_{n}\right) \delta^{3}\left(i \xi_{n}\right)}\left[2{ }^{1} \tilde{\chi}_{1 \perp}^{1+2}\left(0, i \xi_{n}\right)+{ }^{1} \tilde{\chi}_{1 \|}^{1+2}\left(0, i \xi_{n}\right)\left(\delta^{2}\left(i \xi_{n}\right)+1 / 2\right)\right] .
$$


Deux cas limites particulièrement importants peuvent être déduits de cette relation générale :

- Limite quantique : Lorsque les écarts entre les niveaux d'énergie des molécules du système sont grands devant $k_{\mathrm{B}} T$ (c'est le cas des transitions électroniques), la somme $\sum_{n}^{\prime}$ peut être remplacée par l'intégrale $\int_{0}^{+\infty} \mathrm{d} u / 2 \pi k_{\mathrm{B}} T \hbar^{-1}$. Ainsi, dans ce cas, $\mu_{1}\left(z_{0}\right)$ s'écrit :

$$
\mu_{1}\left(z_{0}\right)=-\frac{\hbar}{8 \pi z_{0}^{4}} \int_{0}^{+\infty} \frac{\Delta(i u)}{\bar{\varepsilon}_{1}(i u) \delta^{3}(i u)}\left[{ }^{1} \tilde{\chi}_{1}^{1+2}(0, i u)+{ }^{1} \tilde{\chi}_{1}^{1+2}(0, i u)\left(\delta^{2}(i u)+1 / 2\right)\right] \mathrm{d} u
$$

Ce résultat peut être connecté à l'expression du moment dipolaire induit par adsorption d'un atome sur une surface diélectrique [15]. Pour cela il suffit d'écrire :

- d'une part que l'échantillon contient une seule molécule sphérique.

Les composantes macroscopiques ${ }^{1} \tilde{\chi}_{1 \perp}^{1+2}$ et ${ }^{1} \tilde{\chi}_{1 \|}^{1+2}$ doivent alors être remplacées par les composantes ${ }^{1} \chi_{\perp}^{1+2}$ et ${ }^{1} \chi_{\|}^{1+2}$ du tenseur moléculaire correspondant qui pour la symétrie sphérique vérifie de plus la relation ${ }^{1} \chi_{\|}^{1+2}=\frac{4}{3}{ }^{1} \chi_{\perp}^{1+2} \equiv{ }^{1} \chi^{1+2 z z z z}$;

- d'autre part que le milieu environnant la molécule est le vide $: \bar{\varepsilon}_{1}=\delta=1$.

On retrouve alors un résultat obtenu antérieurement [15] :

$$
\mu=-3 \hbar / 8 \pi z_{0}^{4} \int_{0}^{+\infty}{ }^{1} \chi^{1+2 z z z z}(0, i u)\left(\frac{\varepsilon(i u)-1}{\varepsilon(i u)+1}\right) \mathrm{d} u .
$$

- Limite classique : inversement, si l'on suppose que les intervalles énergétiques dans l'échantillon sont faibles devant $k_{\mathrm{B}} T$ (transitions du type rotationnel) on remarque en examinant les fonctions ${ }^{1} \tilde{\chi}_{1 \perp}^{1+2}\left(0, i \xi_{n}\right)$ et ${ }^{1} \tilde{\chi}_{1 \|}^{1+2}\left(0, i \xi_{n}\right)$ (cf. annexe B) que :

avec

$$
l(0) \gg l\left(i \xi_{1}\right) \gg l\left(i \xi_{2}\right) \ldots
$$

$$
l\left(i \xi_{n}\right)=2{ }^{1} \tilde{\chi}_{1 \perp}^{1+2}\left(0, i \xi_{n}\right)+{ }^{1} \tilde{\chi}_{1 \|}^{1+2}\left(0, i \xi_{n}\right)\left(\delta^{2}\left(i \xi_{n}\right)+1 / 2\right)
$$

de sorte qu'en première approximation, l'équation 32 peut être restreinte au premier terme de la somme, soit :

$$
\mu_{1}\left(z_{0}\right)=-\frac{k_{\mathrm{B}} T}{4 z_{0}^{4}} \frac{\Delta(0)}{\bar{\varepsilon}_{1}(0) \delta^{3}(0)}\left[2{ }^{1} \tilde{\chi}_{1 \perp}^{1+2}(0)+{ }^{1} \tilde{\chi}_{1 \|}^{1+2}(0)\left(\delta^{2}(0)+1 / 2\right)\right]
$$

1.3 Polarisation du milieu (2) isotrope. - Le moment dipolaire de dispersion d'un élément de volume unité situé en $\left(0,0, z_{0}\right)\left(z_{0}<0\right)$ s'obtient par une démarche tout à fait analogue. On a dans ce cas :

où

$$
\mu_{2}\left(z_{0}\right)=\frac{3 k_{\mathrm{B}} T}{8 z_{0}^{4}} \sum_{n}^{\prime}{ }^{1} \tilde{\chi}_{2}^{1+2}\left(0, i \xi_{n}\right) \frac{\Delta\left(i \xi_{n}\right)}{\varepsilon_{2}\left(i \xi_{n}\right)}
$$

\section{Différence de potentiel d'origine dispersive, évaluation numérique.}

On a montré (cf. Eqs. 32 et 37), que tout élément de volume du système est porteur, du fait de la présence d'une interface en $z=0$, d'une polarisation statique $\mu(z)$ variant en $z^{-4}$ où $z$ est la distance entre l'élément de volume et l'interface. Cette distribution de dipôles va engendrer une d.d.p. d'origine dispersive $\Delta \psi$ de part et d'autre de la surface, s'écrivant :

$$
\Delta \psi=4 \pi\left(\int_{-\infty}^{\frac{a_{1}}{2}} \mu_{1}(z) \mathrm{d} z+\int_{\frac{a_{2}}{2}}^{+\infty} \mu_{2}(z) \mathrm{d} z\right) .
$$

Les indices (1) et (2) repèrent respectivement les milieux (1) et (2) et $a_{1}$ et $a_{2}$ sont des longueurs décrivant le caractère non local de la réponse de chacun des milieux (dans la suite ces longueurs pourront être identifiées à 
des diamètres moléculaires appropriés). Ainsi en substituant dans l'équation $38 \mu_{1}(z)$ et $\mu_{2}(z)$ par leurs expressions obtenues dans le cadre de la limite quantique, il vient :

$$
\begin{aligned}
\Delta \psi=-4 \hbar \int_{0}^{+\infty}\left\{\frac{\Delta(i u)}{3 a_{1}^{3} \bar{\varepsilon}_{1}(i u) \delta^{3}(i u)}\left[2{ }^{1} \tilde{\chi}_{1 \perp}^{1+2}(0, i u)+{ }^{1} \tilde{\chi}_{1 \|}^{1+2}(0, i u)\left(\delta^{2}(i u)+1 / 2\right)\right]-\right. \\
\left.-\frac{\Delta(i u)}{a_{2}^{3} \varepsilon_{2}(i u)}{ }^{1} \tilde{\chi}_{2}^{1+2}(0, i u)\right\} \mathrm{d} u .
\end{aligned}
$$

\section{Estimation numérique de $\Delta \psi$.}

Afin d'obtenir un ordre de grandeur de $\Delta \psi$ il est nécessaire tout d'abord d'exprimer dans l'équation 39 les hyperpolarisabilités par unité de volume ${ }^{1} \tilde{\chi}_{1 \perp}^{1+2},{ }^{1} \tilde{\chi}_{1 \|}^{1+2}$ et ${ }^{1} \tilde{\chi}_{2}^{1+2}$ en fonction des tenseurs ${ }^{1} \chi_{1 \perp}^{1+2},{ }^{1} \chi_{1 \|}^{1+2}$ et ${ }^{1} \chi_{2}^{1+2}$ associées aux molécules constituant les deux milieux. Pour établir le lien entre ces deux types de grandeurs, on peut adapter au cas de champs non uniformes le formalisme de Bedeaux et Bloembergen [21, 22] généralisant la correction de type Clausius-Mossotti dans le cadre d'une réponse non linéaire, les détails sont donnés dans l'annexe C. Ainsi en substituant (C.21) et (C.22) dans (39), il vient :

$$
\begin{aligned}
\Delta \psi=- & \frac{4 \hbar}{27} \int_{0}^{+\infty} \Delta(i u)\left\{\rho _ { 1 } \frac { ( \varepsilon _ { 1 \| } ( i u ) + 2 ) ( \varepsilon _ { 1 \| } ( 0 ) + 2 ) } { 3 a _ { 1 } ^ { 3 } \overline { \varepsilon } _ { 1 } ( i u ) \delta ^ { 3 } ( i u ) } \left[2{ }^{1} \chi_{1 \perp}^{1+2}(0, i u)\left(\varepsilon_{1 \perp}(i u)+2\right)+\right.\right. \\
& \left.\left.+{ }^{1} \chi_{1 \|}^{1+2}(0, i u)\left(\varepsilon_{1 \|}(i u)+2\right)\left(\delta^{2}(i u)+1 / 2\right)\right]-\rho_{2} \frac{\left(\varepsilon_{2}(i u)+2\right)^{2}\left(\varepsilon_{2}(0)+2\right)}{a_{2}^{3} \varepsilon_{2}(i u)}{ }^{1} \chi_{2}^{1+2}(0, i u)\right\} \mathrm{d} u .
\end{aligned}
$$

Pour obtenir un ordre de grandeur de $\Delta \psi$, on adoptera pour $\varepsilon(\omega)$ la forme empirique [16]

$$
\varepsilon(\omega)=1+\sum_{a} \frac{C_{a}}{\left(1-i \omega / \omega_{a}\right)}+\sum_{b} \frac{A_{b}}{\left(1-\omega^{2} / \omega^{2} b\right)} .
$$

Nous nous limiterons de plus à une représentation schématique des milieux :

- Le milieu (1) sera représenté par des chaînes d'hydrocarbures saturés disposées perpendiculairement au plan de l'interface. Pour déterminer l'anisotropie de polarisabilité du milieu ainsi constitué, on utilisera certains résultats obtenus par Zwanzig [13] lors d'une étude sur les forces de dispersion entre ces chaînes. En effet, cet auteur a montré, dans le cadre du modèle de Drude-Lorentz, que chaque groupe $\mathrm{CH}_{2}$ considéré comme ayant une polarisabilité isotrope moyenne $\left({ }^{1} \chi_{1}^{1}(0)=1,85 \times 10^{-24} \mathrm{~cm}^{3}\right)$ lorsqu'il est isolé, devient fortement anisotrope une fois inséré dans la chaîne. Ainsi chaque radical $\mathrm{CH}_{2}$ peut être modélisé par un oscillateur harmonique anisotrope présentant les caractéristiques suivantes :

fréquence :

$$
\omega_{1 \|}=0,28 \omega_{1}, \omega_{1 \perp}=1,21 \omega_{1}
$$

polarisabilité statique :

$$
\begin{aligned}
& { }^{1} \chi_{1 \|}^{1}(0)=4,98 \times 10^{-24} \mathrm{~cm}^{3}, \\
& { }^{1} \chi_{1 \perp}^{1}(0)=0,24 \times 10^{-24} \mathrm{~cm}^{3}
\end{aligned}
$$

où $\omega_{1}$ représente la fréquence de l'oscillateur isolé. Nous choisirons $\omega_{1}=1,55 \times 10^{16} \mathrm{rd} / \mathrm{s}$, valeur utilisée dans la référence 16 pour simuler un milieu constitué d'hydrocarbures. Nous pouvons maintenant écrire les fonctions $\varepsilon_{1 \|}(\omega)$ et $\varepsilon_{1 \perp}(\omega)$ sous la forme :

$$
\begin{aligned}
& \varepsilon_{1 \|}(\omega)=1+\frac{4 \pi \rho_{1}{ }^{1} \chi_{1 \|}^{1}(0)\left(\omega_{1 \|}\right)^{2}}{\left(\omega_{1 \|}\right)^{2}-\omega^{2}}, \\
& \varepsilon_{1 \perp}(\omega)=1+\frac{4 \pi \rho_{1}{ }^{1} \chi_{1 \perp}^{1}(0)\left(\omega_{1 \perp}\right)^{2}}{\left(\omega_{1 \perp}\right)^{2}-\omega^{2}} .
\end{aligned}
$$

Par ailleurs, les fonctions hyperpolarisabilités moléculaires ${ }^{1} \chi_{1 \|}^{1+2}(0, \omega)$ et ${ }^{1} \chi_{1 \perp}^{1+2}(0, \omega)$ peuvent être évaluées dans le cadre du modèle de l'oscillateur harmonique anisotrope [25] à partir de leurs formes analytiques données en annexe B (Eq. B.2), on a alors :

$$
\begin{aligned}
& { }^{1} \chi_{1 \|}^{1+2}(0, \omega)=\frac{2}{e_{1}}\left({ }^{1} \chi_{1 \|}^{1}(0)\right)^{2} \frac{\left(\omega_{1 \|}\right)^{2}}{\left(\omega_{1 \|}\right)^{2}-\omega^{2}} \\
& { }^{1} \chi_{1 \perp}^{1+2}(0, \omega)=\frac{3}{2 e_{1}}\left({ }^{1} \chi_{1 \|}^{1}(0)\right)^{2} \frac{\left(\omega_{1 \|}\right)^{2}}{\left(\omega_{1 \perp}\right)^{2}-\omega^{2}}
\end{aligned}
$$

( $e_{1}$ et $\rho_{1}$ représentent la charge fictive des oscillateurs et la densité numérique du milieu (1)).

- Le milieu (2) sera représenté par une assemblée d'oscillateurs harmoniques isotropes ayant pour fréquence $\omega_{2}=1,9 \times 10^{16} \mathrm{rd} / \mathrm{s}$ (bande d'absorption principale u.v. de l'eau). Dans ce cas $\varepsilon_{2}(\omega)$ et ${ }^{1} \chi_{2}^{1+2}(0, \omega)$ peuvent s'écrire :

$$
\begin{gathered}
\varepsilon_{2}(\omega)=1+\frac{\left(n_{2}^{2}-1\right)}{1-\omega^{2} /\left(\omega_{2}\right)^{2}} \\
{ }^{1} \chi_{2}^{1+2}(0, \omega)=\frac{2\left({ }^{1} \chi_{2}^{1}(0)\right)^{2}}{e_{2}} \frac{\omega_{2}^{2}}{\omega_{2}^{2}-\omega^{2}}
\end{gathered}
$$

où $n_{2}=1,327$ est l'indice de réfraction de l'eau dans 
l'u.v. et $e_{2}$ la charge fictive des oscillateurs du milieu (2).

Ainsi en substituant les relations 42 , à 46 dans l'équation 40 , en choisissant pour ${ }^{1} \chi_{2}^{1}(0)$ la valeur caractéristique $1,8 \times 10^{-24} \mathrm{~cm}^{3}$ et pour $e_{1}$ et $e_{2}$ la charge de l'électron, on obtient en intégrant l'équation $40: \Delta \psi=35 \mathrm{mV}$ (M.K.S.A.). Cet ordre de grandeur obtenu à partir d'une représentation assez schématique des milieux (1) et (2), suggère que les effets dispersifs peuvent contribuer de façon non négligeable à la différence de potentiel totale d'une interface. Rappelons en effet à titre de comparaison que les d.d.p. d'origine polaire ou ionique habituellement mesurées en électrochimie peuvent varier entre $\simeq 10 \mathrm{mV}$ et $500 \mathrm{mV}$. Notons également que la valeur ci-dessus est due principalement à la forte anisotropie de polarisabilité du milieu (1) (pour une interface, constituée de deux milieux isotropes de propriétés électriques assez voisins, l'effet dispersif serait beaucoup plus faible, quelques $\mathrm{mV}$ ).

\section{Annexe A.}

TENSEURS DE RANG 2, 3 ET 4 DANS UNE SYMÉTRIE $D_{\infty \mathrm{h}}$ - - Les composantes de toute grandeur physique (vectorielle ou tensorielle) associée à un système matériel, doivent être invariantes par toutes les opérations de recouvrement de ce système. Par conséquent ces quantités $\mathbf{T}$ doivent constituer une base pour la représentation totalement symétrique du groupe en question.

a) Cas d'un tenseur de rang $2: \mathbf{( 2 )}^{\mathbf{T}}$.

Lors d'une rotation $C$, chaque composante de ce tenseur se transforme suivant la loi :

$$
\stackrel{(2)}{T^{i j}}=C^{i l} C^{j k} \stackrel{(2)}{T^{l k}}
$$

c'est-à-dire comme le produit des composantes $A^{i} B^{j}$ de deux vecteurs $\mathbf{A}$ et $\mathbf{B}$. Donc pour étudier les propriétés d'un tenseur de rang 2, on peut le choisir sous la forme $\mathbf{~}^{\mathbf{T}}=\mathbf{A B}$. D'autre part lorsque dans $D_{\infty \mathrm{h}}$ l'axe du référentiel $\mathrm{O} z$ est choisi parallèle à l'axe $C_{\infty}$, les invariants quadratiques qu'il est possible de construire, pour la représentation totalement symétrique, à partir des vecteurs $\mathbf{A}$ et $\mathbf{B}$ sont :

$$
A^{x} B^{x}+A^{y} B^{y} ;\left(A^{x}\right)^{2}+\left(A^{y}\right)^{2} ;\left(B^{x}\right)^{2}+\left(B^{y}\right)^{2} ;\left(A^{z}\right)^{2},\left(B^{z}\right)^{2} \text { et } A^{z} B^{z} .
$$

Parmi ces invariants, seuls $A^{x} B^{x}+A^{y} B^{y}$ et $A^{z} B^{z}$ sont connectés à $\stackrel{(2)}{\mathbf{T}}$ (en $A^{i} B^{j}$ ). Donc au tenseur $\stackrel{(2)}{\mathbf{T}}$, dans la symétrie $D_{\infty \mathrm{h}}$, sont associés les invariants : $T^{x x}+\stackrel{(2)}{T^{y y}}$ et $T^{z z}$.

Considérons maintenant les produits scalaires suivants :

$$
\left(\sum_{i, j}^{(2)} T^{i j} e^{i} e^{j}\right):\left(e^{x} e^{x}+e^{y} e^{y}\right) \quad \text { et } \quad\left(\sum_{i, j}^{(2)} T^{i j} e^{i} e^{j}\right): e^{z} e^{z}
$$

où $e^{i}$ et $e^{j}$ représentent les vecteurs de base du référentiel. La base $\left\{e^{i} e^{j}\right\}$ étant orthonormée, les termes de (A.2)

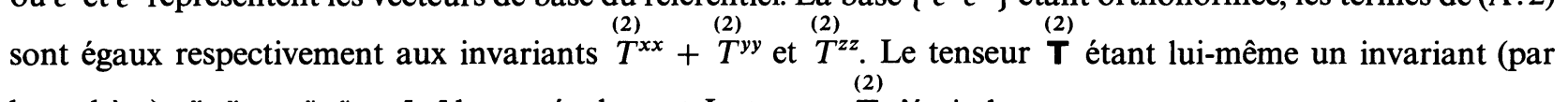
hypothèse), $e^{x} e^{x}+e^{y} e^{y}$ et $e^{z} e^{z}$ le sont également. Le tenseur $\stackrel{(2)}{\mathbf{T}}$ s'écrit donc :

$$
\stackrel{(2)}{\mathbf{T}}=b_{1}\left(e^{x} e^{x}+e^{y} e^{y}\right)+b_{2} e^{z} e^{z}=\sum_{i, j} \stackrel{(2)}{T}^{i j} e^{i} e^{j}
$$

et, par identification, on obtient finalement :

$$
\stackrel{(2)}{T^{z z}}, \stackrel{(2)}{T^{x x}}=\stackrel{(2)}{T^{y y}}
$$

et

$$
\stackrel{(2)}{T^{\alpha \beta}}=0 \quad \text { si } \quad \alpha \neq \beta .
$$

Par exemple, pour le tenseur susceptibilité diélectrique $\varepsilon_{1}$ on retrouve à partir de (A.4) la forme donnée dans l'équation 10.

b) Cas des tenseurs de rang 3.

Le groupe $D_{\infty \mathrm{h}}$ possède un centre d'inversion. Par conséquent, les tenseurs de rang 3 décrivant des propriétés d'un système physique appartenant à ce groupe sont nuls. En particulier, pour les tenseurs hyperpolarisabilité dipolaire champ-champ ${ }^{1} \chi^{1+1}$ et polarisabilité quadrupolaire ${ }^{2} \chi^{1}$, nous avons :

$$
{ }^{1} \chi^{1+1}={ }^{2} \chi^{1}=0 .
$$


c) Cas d'un tenseur de rang 4.

En utilisant la démarche proposée précédemment (cf. $\S$ a)) on montre aisément qu'un tenseur de rang 4 s'écrit dans le groupe $D_{\infty \mathrm{h}}$ avec dix constantes indépendantes $a_{l}(l=1,10)$, soit :

$$
\begin{array}{ll}
\stackrel{(4)}{T}^{x x y y}=\stackrel{(4)}{T}^{y y x x}=a_{1}, & \stackrel{(4)}{T}^{x y x y}=\stackrel{(4)}{T}^{y x y x}=a_{2} \\
\stackrel{(4)}{T}^{x y y x}=\stackrel{(4)}{T}^{y x x y}=a_{3}, & \stackrel{(4)}{T}^{z z z z}=a_{4} \\
\stackrel{(4)}{T}^{x x z z}=\stackrel{(4)}{T}^{y y z}=a_{5}, & \stackrel{(4)}{T}^{x z x z}=\stackrel{(4)}{T}^{y z y z}=a_{6} \\
\stackrel{(4)}{T}^{x z z x}=\stackrel{(4)}{T}^{y z z y}=a_{7}, & \stackrel{(4)}{T}^{z x z x}=\stackrel{(4)}{T}^{z y z y}=a_{8} \\
\stackrel{(4)}{T}^{z x x z}=\stackrel{(4)}{T}_{T}^{z y y z}=a_{9}, & \left(4^{z)}\right)^{z z x x}=\stackrel{(4)}{T}^{z z y y}=a_{10} \\
\stackrel{(4)}{T}^{x x x x}=\stackrel{(4)}{T}_{T}^{y y y}=a_{1}+a_{2}+a_{3} .
\end{array}
$$

Application au tenseur hyperpolarisabilité dipolaire champ gradient de champ ${ }^{1} \chi^{1+2}$ :

Ce tenseur est défini de la façon suivante [23] :

$$
m^{\alpha}\left(\omega_{1}, \omega_{2}\right)=\frac{1}{6}{ }^{1} \chi^{1+2 \alpha, \beta, \gamma \delta}\left(\omega_{1}, \omega_{2}\right) E^{\beta}\left(\omega_{1}\right) \nabla^{\gamma} E^{\delta}\left(\omega_{2}\right) .
$$

Nous pouvons donc écrire puisque $\nabla^{\alpha} E^{\alpha}=0$ et $\nabla^{\gamma} E^{\delta}=\nabla^{\delta} E^{\gamma}$

$$
{ }^{1} \chi^{1+2 \alpha, \beta, \gamma \delta}={ }^{1} \chi^{1+2 \alpha, \beta, \delta \gamma} \quad \text { et } \quad{ }^{1} \chi^{1+2 \alpha, \beta, \gamma \gamma}=0 .
$$

Ces deux conditions supplémentaires permettent de réduire à 5 le nombre de constantes indépendantes prévues initialement (cf. Eq. A.6). On a maintenant :

et

$$
\begin{aligned}
& { }^{1} \chi^{1+2 x x y y}={ }^{1} \chi^{1+2 y y x x}=a_{1} \\
& { }^{1} \chi^{1+2 x y x y}={ }^{1} \chi^{1+2 y x y x}={ }^{1} \chi^{1+2 x y y x}={ }^{1} \chi^{1+2 y x x y}=a_{2} \\
& { }^{1} \chi^{1+2 z z z z}=a_{4},{ }^{1} \chi^{1+2 x z z x}={ }^{1} \chi^{1+2 y z z y}={ }^{1} \chi^{1+2 x z x z}={ }^{1} \chi^{1+2 y z y z}=a_{6} \\
& { }^{1} \chi^{1+2 z x x z}={ }^{1} \chi^{1+2 z y y z}={ }^{1} \chi^{1+2 z x z x}={ }^{1} \chi^{1+2 z y z y}=a_{8} \\
& { }^{1} \chi^{1+2 z z x x}={ }^{1} \chi^{1+2 z z y y}=-\frac{a_{4}}{2}, \quad{ }^{1} \chi^{1+2 x x z z}={ }^{1} \chi^{1+2 y y z z}=-2\left(a_{1}+a_{2}\right) \\
& { }^{1} \chi^{1+2 x x x x}={ }^{1} \chi^{1+2 y y y y}=a_{1}+2 a_{2} .
\end{aligned}
$$

\section{Annexe B.}

EXPRESSION DES TENSEURS ${ }^{1} \chi^{1},{ }^{1} \chi^{1+2}$ ET ${ }^{2} \chi^{1+1}$. - L'expression générale des susceptibilités linéaires et non linéaires est donnée dans la référence 23 . Pour un système dans l'état fondamental ces tenseurs s'écrivent en absence d'amortissement :

$$
{ }^{1} \chi^{1 \alpha \beta}(\omega)=\hbar^{-1} \sum_{r}\left(\frac{\mu_{0 r}^{\alpha} \mu_{r 0}^{\beta}}{\omega_{r 0}+\omega}+\frac{\mu_{0 r}^{\beta} \mu_{r 0}^{\alpha}}{\omega_{r 0}-\omega}\right)
$$

$$
\begin{gathered}
{ }^{1} \chi^{1+2 \alpha, \beta, \gamma \delta}\left(\omega_{a}, \omega_{b}\right)=\hbar^{-2} \sum_{r, s}\left\{\frac{\mu_{0 r}^{\alpha} \mu_{r s}^{\beta} \theta_{s 0}^{\gamma \delta}}{\left(\omega_{r 0}+\omega_{a}+\omega_{0}\right)\left(\omega_{s 0}+\omega_{b}\right)}+\frac{\mu_{0 r}^{\beta} \mu_{r s}^{\alpha} \theta_{s 0}^{\gamma \delta}}{\left(\omega_{r 0}-\omega_{a}\right)\left(\omega_{s 0}+\omega_{b}\right)}+\right. \\
+\frac{\mu_{0 r}^{\beta} \theta_{r s}^{\gamma \delta} \mu_{s 0}^{\alpha}}{\left(\omega_{r 0}-\omega_{a}\right)\left(\omega_{s 0}-\omega_{a}-\omega_{b}\right)}+\frac{\mu_{0 r}^{\alpha} \theta_{r s}^{\gamma \delta} \mu_{s 0}^{\beta}}{\left(\omega_{r 0}+\omega_{a}+\omega_{b}\right)\left(\omega_{s 0}+\omega_{a}\right)}+\frac{\theta_{0 r}^{\gamma \delta} \mu_{r s}^{\alpha} \mu_{s 0}^{\beta}}{\left(\omega_{r 0}-\omega_{b}\right)\left(\omega_{s 0}+\omega_{a}\right)}+ \\
\left.\quad+\frac{\theta_{0 r}^{\gamma \delta} \mu_{r s}^{\beta} \mu_{s 0}^{\alpha}}{\left(\omega_{r 0}-\omega_{b}\right)\left(\omega_{s 0}-\omega_{a}-\omega_{b}\right)}\right\}
\end{gathered}
$$

$$
\begin{array}{r}
{ }^{2} \chi^{1+1 \alpha \beta, \delta, \gamma}\left(\omega_{a}, \omega_{b}\right)=\hbar^{-2} \sum_{r, s}\left\{\frac{\mu_{0 r}^{\gamma} \theta_{r s}^{\alpha \beta} \mu_{s 0}^{\delta}}{\left(\omega_{r 0}-\omega_{a}\right)\left(\omega_{s 0}+\omega_{b}\right)}+\frac{\mu_{0 r}^{\delta} \theta_{r s}^{\alpha \beta} \mu_{s 0}^{\gamma}}{\left(\omega_{r 0}-\omega_{b}\right)\left(\omega_{s 0}+\omega_{a}\right)}+\right. \\
+\frac{\mu_{0 r}^{\gamma} \mu_{r s}^{\delta} \theta_{s 0}^{\alpha \beta}}{\left(\omega_{r 0}-\omega_{a}\right)\left(\omega_{s 0}-\omega_{a}-\omega_{b}\right)}+\frac{\theta_{0 r}^{\alpha \beta} \mu_{r s}^{\gamma} \mu_{s 0}^{\delta}}{\left(\omega_{r 0}+\omega_{a}+\omega_{b}\right)\left(\omega_{s 0}+\omega_{b}\right)}+\frac{\theta_{0 r}^{\alpha \beta} \mu_{r s}^{\delta} \mu_{s 0}^{\gamma}}{\left(\omega_{r 0}+\omega_{a}+\omega_{b}\right)\left(\omega_{s 0}+\omega_{a}\right)}+ \\
\left.\quad+\frac{\mu_{0 r}^{\delta} \mu_{r s}^{\gamma} \theta_{s 0}^{\alpha \beta}}{\left(\omega_{r 0}-\omega_{b}\right)\left(\omega_{s 0}-\omega_{a}-\omega_{b}\right)}\right\} .
\end{array}
$$


Un examen détaillé des composantes des tenseurs ${ }^{1} \chi^{1+2}$ et ${ }^{2} \chi^{1+1}$ montre que :

$$
{ }^{1} \chi^{1+2 \alpha, \beta, \gamma \delta}\left(\omega_{a}, \omega_{b}\right)={ }^{2} \chi^{1+1 \gamma \delta, \alpha, \beta}\left(\omega_{b}, \omega_{a}\right) .
$$

\section{Annexe C.}

RELATION ENTRE LES SUSCEPTIBILITÉS MACROSCOPIQUES NON LINÉAIRES ${ }^{1} \tilde{\chi}^{1+2}\left(\omega_{1}, \omega_{2}\right),{ }^{2} \tilde{\chi}^{1+1}\left(\omega_{1}, \omega_{2}\right)$ ET LES HYPERPOLARISABILITÉS NON LINÉAIRES MOLÉCULAIRES ${ }^{1} \chi^{1+2}\left(\omega_{1}, \omega_{2}\right)$ ET ${ }^{2} \chi^{1+1}\left(\omega_{1}, \omega_{2}\right)$. - Récemment, BedeauX et Bloembergen [21] ont élaboré une formulation générale connectant les susceptibilités dipolaires non linéaires microscopiques et macroscopiques du type ${ }^{1} \chi^{1+1}\left(\omega_{1}, \omega_{2}\right)$ et ${ }^{1} \tilde{\chi}^{1+1}\left(\omega_{1}, \omega_{2}\right)$ dans les diélectriques (relations du type Clausius-Mossotti non linéaire). Nous proposons d'étendre maintenant leur méthode afin d'obtenir des relations du même type entre les tenseurs ${ }^{1} \chi^{1+2}\left(\omega_{1}, \omega_{2}\right)$ et ${ }^{1} \tilde{\chi}^{1+2}\left(\omega_{1}, \omega_{2}\right)$ décrivant une réponse dipolaire en champ et gradient de champ, et entre les tenseurs ${ }^{2} \chi^{1+1}\left(\omega_{1}, \omega_{2}\right)$ et ${ }^{2} \tilde{\chi}^{1+1}\left(\omega_{1}, \omega_{2}\right)$ décrivant une réponse quadrupolaire en champ-champ. Nous nous limiterons toutefois au cas des systèmes possédant un centre d'inversion. (Symétrie sphérique ou $D_{\text {ch }}$.)

C.1 Polarisation du système en fonction des susceptibilités macroscopiques. - La polarisation d'un système matériel développée à l'ordre quadrupolaire s'écrit [24] :

$$
P(r, t)=m(r, t)-1 / 3 \nabla \cdot Q(r, t) .
$$

Les fonctions $m(r, t)$ et $Q(r, t)$ représentent comme précédemment (cf. § 1.1) la polarisation instantanée dipolaire et quadrupolaire du système. Elles s'expriment en fonction du champ macroscopique $E(r, t)$ solution des équations de Maxwell dans un diélectrique sous la forme [23] :

$$
\begin{gathered}
m(r, t)=\int{ }^{1} \tilde{\chi}^{1}\left(t-t_{1}\right) \cdot E\left(r, t_{1}\right) \mathrm{d} t_{1}+\frac{1}{6} \iint\left[{ }^{1} \tilde{\chi}^{1+2}\left(t-t_{1}, t-t_{2}\right) \vdots \nabla E\left(r, t_{1}\right) E\left(r, t_{2}\right)+\right. \\
\left.+{ }^{1} \tilde{\chi}^{2+1}\left(t-t_{1}, t-t_{2}\right) \vdots \nabla E\left(r, t_{1}\right) E\left(r, t_{2}\right)\right] \mathrm{d} t_{1} \mathrm{~d} t_{2} \\
Q(r, t)=\frac{1}{2} \iint{ }^{2} \chi^{1+1}\left(t-t_{1}, t-t_{2}\right): E\left(r, t_{1}\right) E\left(r, t_{2}\right) \mathrm{d} t_{1} \mathrm{~d} t_{2} .
\end{gathered}
$$

C. 2 Polarisation en fonction des grandeurs microscopiques ${ }^{1} \chi^{1},{ }^{1} \chi^{1+2}{ }^{1} \chi^{2+1}$ et ${ }^{2} \chi^{1+1}$ des molécules du système. - Considérons la $l$-ième molécule en $r_{1}$ et dans la configuration angulaire $\theta_{1}$ (angle entre l'axe principale de la molécule et l'axe $z$ ). Dans la matière cette molécule est soumise à un champ électrique $E_{\mathrm{e}}\left(r_{l}, t\right)$ (qui par la suite sera connecté à $E(r, t)$ ). Elle est de plus porteuse d'un dipôle et d'un quadrupôle s'écrivant sous une forme analogue à (C.2) et (C.3), soit :

$$
\begin{gathered}
m_{l}(t)=\int{ }^{1} \chi^{1}\left(t-t_{1} ; \theta_{l}\right) \cdot E_{\mathrm{e}}\left(r_{l}, t_{1}\right) \mathrm{d} t_{1}+\frac{1}{6} \iint{ }^{1} \chi^{1+2}\left(t-t_{1}, t-t_{2}, \theta_{l}\right) \vdots \\
E_{\mathrm{e}}\left(r_{l}, t_{1}\right) \nabla E_{\mathrm{e}}\left(r_{l}, t_{2}\right)+{ }^{1} \chi^{2+1}\left(t-t_{1}, t-t_{2}, \theta_{l}\right) \vdots \nabla E_{\mathrm{e}}\left(r_{l}, t_{1}\right) E_{\mathrm{e}}\left(r_{l}, t_{2}\right) \mathrm{d} t_{1} \mathrm{~d} t_{2} \\
Q_{l}(t)=\frac{1}{2} \iint{ }^{2} \chi^{1+1}\left(t-t_{1}, t-t_{2}, \theta_{l}\right): E_{\mathrm{e}}\left(r_{l}, t_{1}\right) E_{\mathrm{e}}\left(r_{l}, t_{2}\right) \mathrm{d} t_{1} \mathrm{~d} t_{2}
\end{gathered}
$$

avec

$$
\begin{gathered}
{ }^{1} \chi^{1}\left(t-t_{1}, \theta_{l}\right)=R\left(\theta_{l}\right) R\left(\theta_{l}\right){ }^{1} \chi^{1}\left(t-t_{1}, 0\right) \\
{ }^{n} \chi^{p+q}\left(t-t_{1}, t-t_{2}, \theta_{l}\right)=R\left(\theta_{l}\right) R\left(\theta_{l}\right) R\left(\theta_{l}\right) R\left(\theta_{l}\right)^{n} \chi^{n+q}\left(t-t_{1}, t-t_{2}, 0\right)
\end{gathered}
$$

où $R\left(\theta_{l}\right)$ est la matrice de rotation faisant passer la $l$-ième molécule de la configuration angulaire $\theta_{l}=0$ à la configuration $\theta_{l}$, les indices $(n, p, q)$ peuvent prendre les valeurs $(1,1,2),(1,2,1)$ et $(2,1,1) .{ }^{1} \chi^{1}\left(t-t_{1}, 0\right)$, ${ }^{n} \chi^{p+q}\left(t-t_{1}, t-t_{2}, 0\right)$ sont les polarisabilités et hyperpolarisabilités de la molécule dans ses axes principaux. La polarisation du système s'écrit maintenant :

$$
P(r, t)=\sum_{l}\left\{m_{l}\left(r_{l}, t\right)-\frac{1}{3} \nabla \cdot Q\left(r_{l}, t\right)\right\} \delta\left(r-r_{l}\right) .
$$


C. 3 Relation entre le champ $E_{\mathrm{e}}(r, \omega)$ et le champ macroscopique $E(r, \omega)$. - Le champ $E_{\mathrm{e}}$ en un point quelconque du diélectrique s'écrit comme dans la référence 20 , soit :

$$
E_{\mathrm{e}}(r, \omega)=E_{\mathrm{v}}(r, \omega)-4 \pi \sum_{l} H_{\mathrm{d}}\left(r-r_{1}, \omega\right) \cdot m_{l}(\omega)-4 \pi \sum_{l} H_{\mathrm{Q}}\left(\mathbf{r}-\mathbf{r}_{l}, \omega\right) \cdot Q_{l}(\omega)
$$

$E_{\mathrm{v}}(r, t)$ représente le champ en l'absence de matière et $H_{\mathrm{d}}$ et $H_{\mathrm{Q}}$ sont connectés aux propagateurs dipolaire et quadrupolaire $F_{\mathrm{d}}$ et $F_{\mathrm{Q}}$ de la façon suivante :

$$
H_{\lambda}\left(r-r_{l}, \omega\right)=\left\{\begin{array}{l}
F_{\lambda}\left(r-r_{l}, \omega\right) \text { si }\left|r-r_{l}\right|>a \\
\frac{1}{2}\left[F_{\lambda}\left(r-r_{l}, \omega\right)-F_{\lambda}^{+}\left(r-r_{l}, \omega\right)\right] \text { si }\left|r-r_{l}\right|<a,
\end{array}\right.
$$

( $\lambda=\mathrm{d}$ ou $\mathrm{Q}$ et $a$ représente le diamètre moléculaire).

Utilisons maintenant entre propagateur dipolaire et quadrupolaire la relation :

$$
F_{\mathrm{Q}}(r, \omega)=-1 / 3 \nabla_{r} F_{\mathrm{d}}(r, \omega) \text {. }
$$

Le champ effectif s'écrit alors :

$$
E_{\mathrm{e}}(r, \omega)=E_{\mathrm{v}}(r, \omega)-4 \pi \int\left[H\left(r-r^{\prime}, \omega\right) \cdot m\left(r^{\prime}, \omega\right)-\frac{1}{3} \nabla_{r} H\left(r-r^{\prime}, \omega\right): Q\left(r^{\prime}, \omega\right)\right] \mathrm{d} r^{\prime} .
$$

On exprime ensuite $E_{\mathrm{v}}(r, \omega)$ en fonction du champ macroscopique $E(r, \omega)$. Pour cela on écrit que $E(r, \omega)$ est solution de l'équation différentielle :

$$
\nabla \Lambda(\nabla \Lambda E(r, \omega))+\frac{\partial^{2}}{\partial t^{2}} E(r, \omega)=-4 \pi \frac{\partial^{2}}{\partial t^{2}}\left(m(r, \omega)-\frac{1}{3} \nabla_{r} \cdot Q(r, \omega)\right)
$$

dont la solution générale s'écrit :

$$
E(r, \omega)=E_{\mathrm{v}}(r, \omega)-4 \pi \int\left[F_{\mathrm{d}}\left(r-r^{\prime}, \omega\right) \cdot m\left(r^{\prime}, \omega\right)-\frac{1}{3} \nabla_{r} F_{\mathrm{d}}\left(r-r^{\prime}, \omega\right): Q\left(r^{\prime}, \omega\right)\right] \mathrm{d} r^{\prime}
$$

Ainsi en substituant (C.13) dans (C.11) et en posant

on obtient :

$$
G\left(r-r^{\prime}\right)=F\left(r-r^{\prime}, \omega\right)-H\left(r-r^{\prime}, \omega\right)
$$

$$
E_{\mathrm{e}}(r, \omega)=E(r, \omega)+4 \pi \int\left[G\left(r^{\prime}-r, \omega\right) \cdot m\left(r^{\prime}, t\right)-\frac{1}{3} \nabla_{r} G\left(r-r^{\prime}, \omega\right): Q\left(r^{\prime}, t\right)\right] \mathrm{d} r^{\prime}
$$

Il est montré par ailleurs [21] que pour des molécules sphériques, on a : $\lim _{\mathbf{a} \rightarrow 0} G\left(r^{\prime}-r, \omega\right)=1 / 3 \delta\left(r^{\prime}-r\right)$. On supposera ici qu'il est encore possible d'appliquer cette relation à des molécules allongées. Il vient alors :

$$
E_{\mathrm{e}}(r, \omega)=E(r, \omega)+\frac{4 \pi}{3} P(r, \omega)
$$

C. 4 Connexion entre tenseurs macroscopiques et microscopiques. - La polarisation définie par la relation (C.7) doit être moyennée (barre horizontale) sur toutes les positions $r_{l}$ et les orientations $\theta_{l}$ des molécules du système. Ainsi, en négligeant comme dans la référence 21 toutes corrélations entre $r_{l}$ et $\theta_{l}$, on peut écrire, compte tenu de l'équation C. 15 :

$$
\overline{P(r, \omega)}=\rho\left\{\overline{{ }^{1} \chi^{1}(\omega, \theta)} .(E(r, \omega)+4 \pi / 3 \overline{P(r, \omega)})+\text { T.F. }(\omega)\left[\overline{P_{\mathrm{NL}}(r, t)}\right]\right\}
$$

où $\rho$ représente la densité numérique du système, le deuxième terme du membre de droite est la transformée de Fourier temporelle des termes non linéaires contenus dans les équations C. 4 et C.5. Regroupons maintetenant les termes en $\overline{P(r, \omega)}$ dans le membre de gauche de (C.16); il vient alors :

$$
\overline{P(r, \omega)}=\rho\left[1-\frac{4 \pi}{3} \rho \overline{{ }^{1} \chi^{1}(\omega, \theta)}\right]^{-1} \cdot \overline{{ }^{1} \chi^{1}(\omega, \theta)} . E(r, \omega)+\left[1-\frac{4 \pi}{3} \rho^{1 \chi^{1}(\omega, \theta)}\right]^{-1} \text { T.F. }(\omega)\left[\overline{P_{\mathrm{NL}}(r, t)}\right]
$$


On identifie tout d'abord les parties linéaires en $E(r, \omega)$ dans (C. 17) et (C. 1) et on obtient ainsi la relation de Clausius-Mossotti linéaire :

$$
{ }^{1} \tilde{\chi}^{1}(\omega) \equiv \varepsilon(\omega)-1=\rho\left[1-\frac{4 \pi}{3}{ }^{1} \chi^{1} \overline{(\omega, \theta)}\right]^{-1}
$$

ou encore

$$
\left[1-\frac{4 \pi}{3} \overline{{ }^{1} \chi^{1}(\omega, \theta)}\right]^{-1}=\frac{1}{3}[\varepsilon(\omega)+2] .
$$

De même, pour les termes non linéaires, on peut écrire, après identification :

$$
\begin{aligned}
& \frac{\rho}{18}(\varepsilon(\omega)+2) \cdot\left[\overline{\left[\chi^{1+2}\left(\omega-\omega^{\prime}, \omega^{\prime}, \theta\right)\right.} \vdots E_{\mathrm{e}}\left(\omega-\omega^{\prime}, r\right) \nabla E_{\mathrm{e}}\left(\omega^{\prime}, r\right)+\right. \\
& +\overline{{ }^{1} \chi^{2+1}\left(\omega-\omega^{\prime}, \omega^{\prime}, \theta\right)} \nabla E_{\mathrm{e}}\left(\omega-\omega^{\prime}, r\right) E_{\mathrm{e}}\left(\omega^{\prime}, r\right) \\
& \left.+\nabla \cdot \overline{{ }^{2} \chi^{1+1}\left(\omega-\omega^{\prime}, \omega^{\prime}, \theta\right)}: E_{\mathrm{e}}\left(\omega-\omega^{\prime}, r\right) E_{\mathrm{e}}\left(\omega^{\prime}, r\right)\right] \\
& =\frac{1}{6}\left[{ }^{1} \tilde{\chi}^{1+2}\left(\omega-\omega^{\prime}, \omega^{\prime}\right) \vdots E\left(\omega-\omega^{\prime}, r\right) \nabla E\left(\omega^{\prime}, r\right)+{ }^{1} \tilde{\chi}^{2+1}\left(\omega-\omega^{\prime}, \omega^{\prime}\right) \vdots \nabla E\left(\omega-\omega^{\prime}, r\right) E\left(\omega^{\prime}, r\right)\right. \\
& \left.-\nabla^{2} \tilde{\chi}^{1+1}\left(\omega-\omega^{\prime}, \omega^{\prime}\right): E\left(\omega-\omega^{\prime}, r\right) E\left(\omega^{\prime}, r\right)\right] \text {. }
\end{aligned}
$$

Et finalement en substituant $E_{\mathrm{e}}(r, t)$ par son expression au premier ordre issue de la relation C.15, on obtient entre chaque famille de tenseurs les relations suivantes :

$$
\begin{aligned}
& { }^{1} \tilde{\chi}^{1+2 \alpha, \beta, \gamma \delta}\left(\omega_{a}, \omega_{b}\right)=\left[\frac{\rho}{27} \varepsilon\left(\omega_{a}+\omega_{b}\right)+2\right]^{\alpha i}\left[\varepsilon\left(\omega_{a}\right)+2\right]^{i \beta}\left[\varepsilon\left(\omega_{b}\right)+2\right]^{k \delta} \overline{{ }^{1} \chi^{1+2 i j \gamma k}\left(\omega_{a}, \omega_{b}, \theta\right)} \\
& { }^{1} \tilde{\chi}^{2+1 \alpha, \beta, \gamma \delta}\left(\omega_{a}, \omega_{b}\right)=\frac{\rho}{27}\left[\varepsilon\left(\omega_{a}+\omega_{b}\right)+2\right]^{\alpha i}\left[\varepsilon\left(\omega_{a}\right)+2\right]^{\gamma j}\left[\varepsilon\left(\omega_{b}\right)+2\right]^{\delta k} \overline{{ }^{1} \chi^{2+1 i \beta j k}\left(\omega_{a}, \omega_{b}, \theta\right)} \\
& { }^{2} \tilde{\chi}^{1+1 \alpha \beta, \gamma, \delta}\left(\omega_{a}, \omega_{b}\right)=\frac{\rho}{27}\left[\varepsilon\left(\omega_{a}+\omega_{b}\right)+2\right]^{\alpha i}\left[\varepsilon\left(\omega_{a}\right)+2\right]^{j \gamma}\left[\varepsilon\left(\omega_{b}\right)+2\right]^{k \delta} \overline{{ }^{2} \chi^{1+1 i \beta j k}\left(\omega_{a}, \omega_{b}, \theta\right)}
\end{aligned}
$$

C. 5 Application au calcul des composantes ${ }^{1} \chi_{1 \perp}^{1+2}(0, i u),{ }^{1} \chi_{1 \|}^{1+2}(0, i u)$ et ${ }^{1} \chi_{2}^{1+2}(0, i u)$. - Précédemment, nous avions posé :

$$
{ }^{1} \tilde{\chi}_{1 \perp}^{1+2}(0, i u)={ }^{1} \tilde{\chi}_{1}^{1+2 x z x z}(0, i u), \quad{ }^{1} \tilde{\chi}_{1 \|}^{1+2}(0, i u)={ }^{1} \tilde{\chi}_{1}^{1+2 z z z z}(0, i u) \quad \text { et } \quad{ }^{1} \tilde{\chi}_{2}^{1+2}(0, i u)={ }^{1} \tilde{\chi}_{2}^{1+2 z z z z}(0, i u) .
$$

Les relations C. 20 et C. 6 permettent maintenant d'exprimer ces composantes caractérisant les propriétés macroscopiques du système étudié en fonction des composantes des tenseurs hyperpolarisabilité des molécules des milieux (1) et (2), soit :

- Milieu (1) :

$$
\begin{aligned}
& { }^{1} \tilde{\chi}_{1 \|}^{1+2}(0, i u)=\frac{\rho_{1}}{27}\left[\varepsilon_{1 \|}(i u)+2\right]\left[\varepsilon_{1 \|}(i u)+2\right]\left[\varepsilon_{1 \|}(0)+2\right] \overline{R^{z \alpha}(\theta) R^{z \beta}(\theta) R^{z \gamma}(\theta) R^{z \delta}(\theta)}{ }^{1} \chi_{1}^{1+2 \alpha, \beta, \gamma \delta}(0, i u) \\
& { }^{1} \tilde{\chi}_{1 \perp}^{1+2}(0, i u)=\frac{\rho_{1}}{27}\left[\varepsilon_{1 \perp}(i u)+2\right]\left[\varepsilon_{1 \|}(0)+2\right]\left[\varepsilon_{1 \|}(i u)+2\right] \overline{R^{x \alpha}(\theta) R^{z \beta}(\theta) R^{x \gamma}(\theta) R^{z \delta}(\theta)}{ }^{1} \chi_{1}^{1+2 \alpha \beta \gamma \delta}(0, i u)
\end{aligned}
$$

Si l'on suppose de plus que les fluctuations d'orientation des molécules du milieu (1) autour de la direction $\theta=0$ sont de très faible amplitude (cas limite $T \rightarrow 0$ ) alors, la matrice de rotation $R(\theta)$ peut être identifiée à la matrice unité. On peut alors remplacer dans (C.21) la moyenne $\overline{R^{i \alpha}(\theta) R^{i \beta}(\theta) R^{k \delta}(\theta) R^{l \delta}(\theta)}$ par $\delta^{i \alpha} \delta^{j \beta}$ $\delta^{k \delta} \delta^{l \delta}$.

- Milieu (2) isotrope :

Les molécules de ce milieu étant supposées de forme sphérique, il n'y a pas d'effet d'orientation. Par conséquent on peut écrire la composante ${ }^{1} \tilde{\chi}_{2}^{1+2}(0, i u)$ sous la forme :

$$
{ }^{1} \tilde{\chi}_{2}^{1+2}(0, i u)=\frac{\rho_{2}}{27}\left[\varepsilon_{2}(i u)+2\right]^{2}\left[\varepsilon_{2}(0)+2\right]{ }^{1} \chi_{2}^{1+2}(0, i u)
$$

avec

$$
{ }^{1} \chi_{2}^{1+2}(0, i u) \equiv{ }^{1} \chi_{2}^{1+2 z z z z}(0, i u) .
$$




\section{Bibliographie}

[1] London, F., Z. Phys. 63 (1930) 245.

[2] London, F., Z. f. Phys. Chem. B 11 (1930) 222.

[3] Lifchitz, E: M., J. Exper. Theoret. Phys. USSR 29 (1955) 94.

[4] LifchitZ, E. M., Sov. Phys. JETP 2 (1956) 73.

[5] Van Kampen, N. G., Nijboer, B. R. A., Schram, S., Phys. Letters 26A (1968) 387.

[6] Gerlach, E., Phys. Rev. B 4 (1971) 393.

[7] Agarwal, G. S., Phys. Letters 43A (1973) 447.

[8] Galatry, L., Gharbi, T., Chem. Phys. Letters 75 (1980) 427.

[9] Craig, D. P., Thirunamachandran, T., Chem. Phys. Letters 80 (1981) 14.

[10] Hunt, K. L. C., Zilles, B. A., Bohr, J. E., J. Chem. Phys. 75 (1981) 3079.

[11] Galatry, L., Gharbi, T., J. Chem. Phys. 75 (1981) 3608.

[12] Girard, C., Galatry, L., Chem. Phys. Letters 87 (1982) 432.

[13] Zwanzig, R., J. Chem. Phys. 39 (1963) 2251.
[14] Richmond, P., Sarkies, K. W., J. Phys. C 6 (1973) 401.

[15] Galatry, L., Girard, C., Mol. Phys. 49 (1983) 1085.

[16] Mahanty, J., Ninham, B. W., Dispersion Forces (Academic Press, London) 1976.

[17] Landau, L., Lifchitz, E. M., Electrodynamics of Continuous Media (Pergamon London) 1960.

[18] Agarwal (G. S.), Phys. Rev. A 11 (1975) 230.

[19] Parsegian, V. A., Weiss, H. G., J. Adhesion 3 (1972) 259.

[20] Agarwal, G. S., Pattanayak, D. N., Wolf, E., Phys. Rev. B 10 (1974) 1447;

Maradudin, A. A., Mills, D. L., Phys. Rev. B 7 (1973) 2787.

[21] Bedeaux, D., Bloembergen, N., Physica 69 (1973) 57.

[22] Bedeaux, D., Mazur, P., Physica 67 (1973) 23.

[23] Kielich, S., Acta Phys. Polon. 29 (1966) 875.

[24] DE GroOt, S. R., The Maxwell equations (North Holland Publishing Company, Amsterdam) 1969.

[25] Girard, C., Thèse 3e cycle, Besançon, 1982. 